\title{
An $h$-Adaptive Finite Element Solution of the Relaxation Non-Equilibrium Model for Gravity-Driven Fingers
}

\author{
Huanying Bian ${ }^{1}$, Yedan Shen ${ }^{1}$ and Guanghui $\mathrm{Hu}^{1,2, *}$ \\ ${ }^{1}$ Department of Mathematics, Faculty of Science and Technology, University of Macau, \\ Macao SAR, China \\ 2 Zhuhai UM Science \& Technology Research Institute, Zhuhai, Guangdong 519031, \\ China
}

Received 18 July 2020; Accepted (in revised version) 8 December 2020

\begin{abstract}
The study on the fingering phenomenon has been playing an important role in understanding the mechanism of the fluid flow through the porous media. In this paper, a numerical method consisting of the Crank-Nicolson scheme for the temporal discretization and the finite element method for the spatial discretization is proposed for the relaxation non-equilibrium Richards equation in simulating the fingering phenomenon. Towards the efficiency and accuracy of the numerical simulations, a predictor-corrector process is used for resolving the nonlinearity of the equation, and an $h$-adaptive mesh method is introduced for accurately resolving the solution around the wetting front region, in which a heuristic a posteriori error indicator is designed for the purpose. In numerical simulations, a traveling wave solution of the governing equation is derived for checking the numerical convergence of the proposed method. The effectiveness of the $h$-adaptive method is also successfully demonstrated by numerical experiments. Finally the mechanism on generating fingers is discussed by numerically studying several examples.
\end{abstract}

AMS subject classifications: 65M60

Key words: Non-equilibrium Richard equation, $h$-adaptive mesh method, a posteriori error estimation, fingering phenomenon, porous media flow.

\section{Introduction}

To understand the mechanism of the preferential flow has been an important topic in the research area of the porous media flows. For example, in the case of infiltration of water in soil, the filtering capacity of the soil will be lost when infiltration occurs through a few

${ }^{*}$ Corresponding author.

Email: garyhu@um.edu.mo (G. Hu) 
preferential flow paths, which may cause serious disaster [29]. Several factors have been found for the formation of the preferential flow. See $[7,21]$ for a comprehensive review.

There have been a number of works on understanding the mechanism of the formation of preferential flows. In one dimensional case, a phenomenon called saturation overshoot or pressure overshoot has been studied in detail [7,21]. From the plenty of the physical experiments, it has been found that the saturation overshoot caused the instability of the porous media flow [6]. Such conclusion has been successfully confirmed from the linear stability analysis based on non-equilibrium Richards equation [10,22]. In two dimensional case, the hold-back-pile-up effect [11] has been found to be an important mechanism for the formation of preferential flows. The hold-back operates at the forward edge of the wetting front to prevent overspreading due to the capillary diffusion, while the pile-up operates behind to increase the pressure and the water saturation of a finger tip [7].

Many models have been provided in the market for describing the porous media flows, from which the following two principal features can be concluded, i.e., i) the capability of generating initial unstable growth of small perturbations of the wetting front, and ii) the ability to limit lateral spreading behind the unstable front to keep the initially growing perturbations [10,21]. Due to its unconditional stability [10], the Richards equation (RE) can not be an appropriate model for describing the preferential flow. The relaxation non-equilibrium Richards equation (NERE) proposed in [15] is able to produce truly non-monotonic saturation profiles [9], by taking the dynamic (relaxation) effects with non-equilibrium pressure-saturation relation into account. Hassanizadeh et al. also showed in [20] that the hysteresis in the capillary pressure-saturation relation was a key point on the persistence of the fingers. In a number of physical experiments, the hysteresis phenomenon has been successfully observed, which describes a special relation between pressure and saturation. Consequently, in [2], Beliaev and Hassanizadeh developed a new model of capillary hysteresis by combining the hysteresis memory effects and dynamic (relaxation) effects together, and Schweizer [25] showed the mechanism and the importance of hysteresis in the study of preferential flows.

Towards the numerical methods for simulating porous media flows, a number of pioneering works have been available in the literature, for example, the finite difference methods [3,21,32,33], the discontinuous Galerkin methods [26], the finite element methods $[16,17,24,30,34]$, etc. Besides, a convolutional neural network [12] is developed for rapidly predicting multiphase flow in heterogeneous porous media. For the profile of the finger, the water saturation around the finger tip is higher than that in the stationary finger core and a distribution layer [21]. Based on this observation, a uniformly fine mesh is not a smart choice for an efficient simulation. To balance the computational resources and the numerical accuracy, a discretization of the governing equation on a dynamically nonuniform mesh becomes a feasible way, which can be realized by using the adaptive mesh methods. The adaptive mesh techniques mainly include the $r$-adaptive methods [19] which redistribute the grid points while keeping the total number of mesh grids unchanged, the $h$-adaptive methods which locally refine and coarsen the mesh, and the 
$p$-adaptive methods which locally enrich the order of the basis functions. Dong et al. [8] showed that moving mesh method could be an effective way to approximate two-phase flows in porous media. Zegeling et al. [31] investigated non-equilibrium PDE model with an adaptive moving mesh method, which successfully showed that for the same accuracy, the adaptive method needed around a factor of four fewer grid points than the uniform grid case. Based on the outstanding advantages of moving mesh method in tracking shocks or steep wave fronts of other two-phase flow equations [8,16,33], Zhang and Zegeling presented an efficient mixed finite element method [34] to solve the fourthorder thin film flow equations using moving mesh method. Lamacz et al. [17] proposed a Galerkin scheme for hysteresis model, which showed the convergence of the corresponding approximate solutions and the existence of weak solutions, and adopted the $h$-adaptive strategy to show the effect of gravity driven fingering in porous media. The NERE model has been solved by a $r$-adaptive method [16].

In this paper, we propose an $h$-adaptive finite element method to solve the NERE. The method can be summarized as follows. The numerical discretization of the NERE consists of the finite element method for the spatial discretization and the Crank-Nicolson $(\mathrm{CN})$ method for the temporal discretization. To handle the nonlinearity of the governing equation, a predictor-corrector approach is applied, in which a forward Euler method is used for the solution prediction, while several correction steps are implemented based on $\mathrm{CN}$ method to improve the accuracy of the solution. To enhance the simulation efficiency, an $h$-adaptive mesh method is introduced to dynamically control the total amount of the mesh grids. Several linear systems need to be solved in each predictor-corrector procedure since the finite element discretization is used for the spatial discretization, and an efficient algebraic multigrid solver is employed for efficiently solving the linear equations.

The efficient implementation of the $h$-adaptive mesh method for a temporal problem depends on three quality components, i.e., a tool for handling the local refinement and/or coarsening of the mesh grids, a posteriori error estimation based on the numerical solutions, and the interpolation of the numerical solutions between two meshes. First of all, we adopt the efficient hierarchy geometry tree (HGT) from [18] to handle the local refinement and/or coarsening of the mesh. Secondly, a heuristic a posteriori error estimator is designed with the following three parts, i.e., the jump of the gradient of the saturation across the element edges, the residual of the governing equation in each element, as well as the solution difference between two adjacent time instants. Thirdly, it can be observed that with the HGT, there is a belonging-to relation for two elements from two meshes, respectively. Then the efficient implementation on the solution interpolation can be expected based on this belonging-to relation.

For the combination of the $h$-adaptive mesh method and the predictor-corrector process, towards minimizing the total amount of the mesh grids, one straightforward approach is to implement the local refinement and/or coarsening of the mesh grids in each prediction or correction steps. With this approach, the mesh grids will always be tailored for the updated solution. However, the potential issue for such strategy is the 
efficiency of the simulation, since for each implementation of the adaptive refinement of the mesh grids, there are associated interpolation operation for updating the solution. Furthermore, with the fluctuation of the wetting front with the time evolution, frequent implementations of the $h$-adaptive method would cause repeated operations between the refinement and coarsening in some region around the wetting front within a short time period. Hence, the following algorithm is designed to avoid the above issues. First of all, in each predictor-corrector process, the $h$-adaptive method only be implemented after the prediction step. In addition, we only add new mesh grids during this process, which means that no grid point will be removed from the mesh. Secondly, a complete $h$-adaptive process, including the mesh refinement and coarsening, will be implemented for every several time propagation steps. With above strategy, the old solution in each predictor-corrector process can always be hold accurately, while the repeated operations can be effectively avoided. The numerical experiments successfully show the numerical convergence of the proposed $h$-adaptive finite element method, and the ability on resolving the profile of the finger with less mesh grids, compared with the results from the uniform meshes. With the proposed method, the dynamics on the propagation of the wetting front, as well as the formation of the fingers governed by NERE model are also studied.

The paper is organized as follows. In Section 2, the NERE model is reviewed and the effect of relaxation coefficient $\tau$ on the stability of NERE is studied. In Section 3, we first give the spatial and temporal discretizations of the NERE model, and then describe an $h$-adaptive method. The design of the error indicator, and the flowchart of the algorithm are also described in this section. In Section 4, the numerical experiments are carried out and analyzed. Finally, the conclusion of this paper and future work are given in Section 5 .

\section{The relaxation non-equilibrium Richards equation}

\subsection{Governing equation}

Assuming that the porosity $\phi$ of the medium is a constant, the fluid mass balance equation for unsaturated porous media reads

$$
\phi \frac{\partial \rho \Theta}{\partial t^{*}}+\nabla \cdot(\rho \mathbf{q})=0
$$

and the Darcy's Law $[1,23]$ reads

$$
\mathbf{q}=-\frac{\kappa}{\mu} \nabla(p+\rho g z)
$$

where $\mathbf{q}$ denotes the fluid volumetric flux vector $\left[\mathrm{m}^{3} /\left(\mathrm{m}^{2} \cdot s\right)\right], t^{*}$ time $[s], \rho$ the fluid density $\left[\mathrm{kg} / \mathrm{m}^{3}\right], g$ the gravitational acceleration $\left[\mathrm{m} / \mathrm{s}^{2}\right], \Theta$ the fluid saturation $\left[\mathrm{m}^{3} / \mathrm{m}^{3}\right], p$ the fluid pressure $\left[\mathrm{N} / \mathrm{m}^{2}\right], \mu$ the dynamic viscosity $[\mathrm{kg} /(\mathrm{m} \cdot \mathrm{s})], z$ the $\mathbf{z}$-axis, pointing upward, 
and $\kappa$ the intrinsic permeability tensor $\left[\mathrm{m}^{2}\right]$. Assuming that the mobility and compressibility of air are much larger than those of water [5,23], the mass balance equation can be written as the volumetric balance equation

$$
\frac{\partial \theta}{\partial t^{*}}=\nabla^{*} \cdot\left(k^{*}(\theta) \nabla^{*}\left(\hat{\Psi}^{*}+z^{*}\right)\right)
$$

where $\theta=\phi \Theta$ denotes the volumetric water content, $k^{*}(\theta)=\kappa \rho g / \mu$ hydraulic conductivity $[m / s]$, and $\hat{\Psi}^{*}=p /(\rho g)$ the pressure head $[m]$. In [14], Hassanizadeh and Gray advocated a non-equilibrium version of the pressure-saturation relationship, which reflected the rate of change of saturation to the difference between the dynamic water pressure and the saturation-dependent equilibrium water pressure,

$$
\frac{\tau^{*}}{\rho g} \frac{\partial \theta}{\partial t^{*}}=\hat{\Psi}^{*}-\Psi^{*}(s)
$$

where $\tau^{*}$ is a suitably chosen capillary relaxation function $[\mathrm{kg} /(\mathrm{m} \cdot \mathrm{s})], \Psi^{*}(\mathrm{~s})$ represents a dynamic equilibrium water pressure head $[m]$. By setting $x=\alpha_{w}^{*} x^{*}, z=\alpha_{w}^{*} z^{*}, \Psi=\alpha_{w}^{*} \Psi^{*}$, $\hat{\Psi}=\alpha_{w}^{*} \hat{\Psi}^{*}$, hydraulic conductivity $K(s)=k^{*} / k_{0}, t=\alpha_{w}^{*} k_{0} t^{*} /\left(\theta_{s}-\theta_{r}\right)$ and effective saturation $s=\left(\theta-\theta_{r}\right) /\left(\theta_{s}-\theta_{r}\right), 0 \leq \theta_{r} \leq \theta \leq \theta_{s} \leq \phi$, we rewrite Eqs. (2.3) and (2.4) in dimensionless form $[3,5,23]$ as follows

$$
\frac{\partial s}{\partial t}=\nabla \cdot(K(s) \nabla(\hat{\Psi}+z)),
$$

where $k_{0}$ is the fully saturated value at $s=1,\left(\alpha_{w}^{*}\right)^{-1}$ (the reciprocal of the van Genuchten parameter for the main wetting curve) has been used as the natural length scale

$$
\tau \frac{\partial s}{\partial t}=\hat{\Psi}-\Psi(s)
$$

where $\tau=\left(\alpha_{w}^{*}\right)^{2} k_{0} \tau^{*} /(\rho g)>0$ denotes the dimensionless relaxation coefficient function as a positive constant. Hence our governing equation can be written as

$$
\frac{\partial s}{\partial t}=\nabla \cdot\left(K(s) \nabla\left(\tau \frac{\partial s}{\partial t}+\Psi(s)+z\right)\right)
$$

with the following initial and boundary conditions

$$
\left\{\begin{array}{l}
s(x, z, 0)=s_{\text {init }}, \\
\left.\nabla s \cdot \vec{n}\right|_{\partial \Omega_{N}}=0, \\
\left.s(x, z, t)\right|_{\partial \Omega_{D}}=s_{0} .
\end{array}\right.
$$

Here $\Omega$ represents the computational domain, $\partial \Omega_{N}$ and $\partial \Omega_{D}$ represent the Neumann boundary and Dirichlet boundary, respectively. $s_{\text {init }}$ is the initial saturation function on $\Omega$ at $t=0, \vec{n}$ is the unit outward normal vector, $s_{0}$ is the saturation distribution on the upper boundary. See Fig. 1 for the description of computational domain and corresponding boundary conditions. 


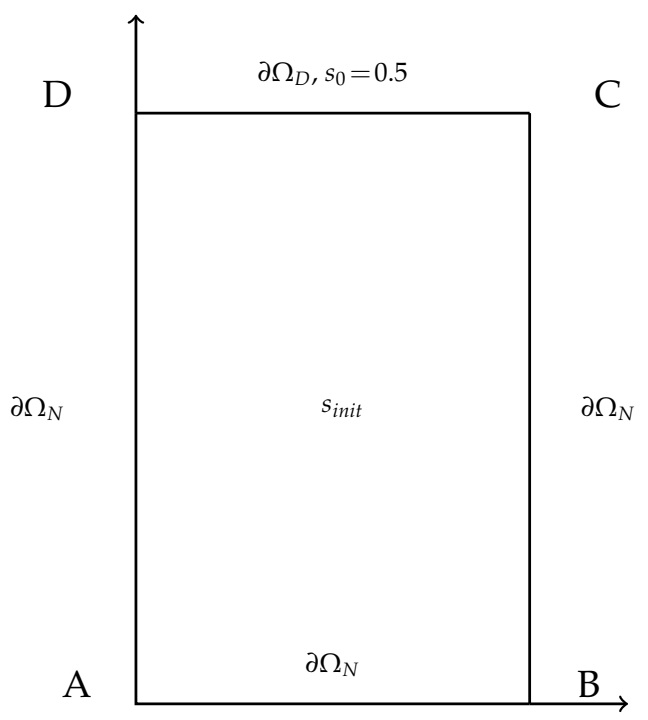

Figure 1: The computational region and the boundary condition of NERE model.

\subsection{Stability on 1D NERE}

It is known that with appropriate parameters, NERE will generate the non-monotone profile of the saturation, i.e., an over saturated tip would be generated around the wetting front, following by the fluctuation on the saturation. The selection of the parameters for this phenomenon can be summarized by the following analysis.

By substituting (2.6) into (2.5), we can obtain the governing equation as follows

$$
\frac{\partial s}{\partial t}=\nabla \cdot \mathcal{D}(s) \nabla s+\frac{\partial K(s)}{\partial z}+\tau \nabla \cdot\left(K(s) \nabla\left(\frac{\partial s}{\partial t}\right)\right)
$$

where $D(s)=K(s) \Psi^{\prime}(s)$ is the diffusivity function, the prime means the first derivative with respect to the saturation $s$.

The traveling wave solution of Eq. (2.9) can be found by using the following approach, in 1-D case. Assuming that a positive constant speed $v$ meets traveling wave coordinate $\xi=z+v t$, and substituting $s(z, t)=u(\xi), \xi \in \mathcal{R}$, into Eq. (2.9), we arrive at the third-order non-linear ODE

$$
v \frac{d u}{d \xi}=\frac{d}{d \xi}\left(\mathcal{D}(u) \frac{d u}{d \xi}\right)+\frac{d K(u)}{d \xi}+v \tau \frac{d}{d \xi}\left(K(u) \frac{d^{2} u}{d \xi^{2}}\right)
$$

Integrating (2.10) in both sides by using $s_{-}:=u(-\infty)$ and the vanished derivatives at 
infinity, we can get the following system by introducing a new variable $\mathcal{H}$,

$$
\left\{\begin{array}{l}
\frac{d u}{d \xi}=\mathcal{H}, \\
\tau v K(u) \frac{d \mathcal{H}}{d \xi}=-D(u) \mathcal{H}+v\left(u-s_{-}\right)+K\left(s_{-}\right)-K(u) .
\end{array}\right.
$$

By taking the limit $\xi \rightarrow+\infty$, we can obtain an asymptotic expression for the wave speed $v=\left(K\left(s_{+}\right)-K\left(s_{-}\right)\right) /\left(s_{+}-s_{-}\right)$by using $s_{+}:=u(+\infty)$.

There are only two critical points $\left(s_{-}, 0\right)$ and $\left(s_{+}, 0\right)$ in the phase plane $(u, \mathcal{H})$ of system (2.11). To determine the characters of the two points, we need to linearize the nonlinearity of the system (2.11). Then the eigenvalues are given as follows

$$
\lambda_{-,+}=-\frac{D\left(s_{0}\right)}{2 v \tau K\left(s_{0}\right)} \pm \sqrt{\Delta}, \quad \Delta=\frac{1}{4}\left(\frac{D\left(s_{0}\right)}{v \tau K\left(s_{0}\right)}\right)^{2}+\frac{v-K^{\prime}\left(s_{0}\right)}{v \tau K\left(s_{0}\right)},
$$

where $s_{0}$ represents $s_{-}$or $s_{+}$. For the point $\left(s_{-}, 0\right)$, the product of $\lambda_{-}$and $\lambda_{+}$is negative, $\Delta>0$, which implies that the point $\left(s_{-}, 0\right)$ is an unstable saddle point. For the point $\left(s_{-}, 0\right)$, the sign of discriminant $\Delta$ is up to a critical parameter

$$
\tau_{c r i}=\frac{\left(D\left(s_{+}\right)\right)^{2}}{4 v\left(K^{\prime}\left(s_{+}\right)-v\right) K\left(s_{+}\right)} .
$$

When $\tau>\tau_{c r i}, \lambda_{-}, \lambda_{+}$are complex numbers, which implies that the point $\left(s_{+}, 0\right)$ is a stable focus(spiral point). Otherwise $\lambda_{-}, \lambda_{+}$are real numbers with $\lambda_{-} \lambda_{+}>0$, which implies that the point $\left(s_{+}, 0\right)$ is a stable node. Hence, traveling wave solutions for (2.10) in phase plane $(u, \mathcal{H})$ connect an unstable critical point $\left(s_{-}, 0\right)$ with a stable one $\left(s_{+}, 0\right)$.

To verify the theoretical results, several numerical experiments have been done by using the parameters $D(u)=0.4, K(u)=u^{2}, s_{+}=0.5$, and $s_{-}=0.01$. By fourth-order Runge-Kutta schemes, we give the corresponding 1-D pseudo-analytical wave solutions, which showed in Fig. 2, for different $\tau$. According to the theoretical analysis, we get $\tau_{c r i} \approx 0.64$, which means that when the non-equilibrium coefficient parameter $\tau>\tau_{c r i}$, the profile of the saturation becomes non-monotone. This is confirmed very well by our numerical results. In Fig. 2, when $\tau=0.5$, which is smaller than $\tau_{c r i}$, a monotone profile of the saturation is observed well. When $\tau=0.8$, the profile becomes non-monotone. In addition, the value of the saturation is bigger than $s_{+}$, which means the appearance of the saturation overshoot. Our results agree well with those presented in $[3,16]$.

\section{The $h$-adaptive finite element method for NERE model}

In this section, we will introduce a numerical framework to solve the NERE model. The framework consists of the finite element method for the spatial discretization, and the Crank-Nicolson method for the temporal discretization. An $h$-adaptive method is introduced to resolve the efficiency issue of the algorithm. 


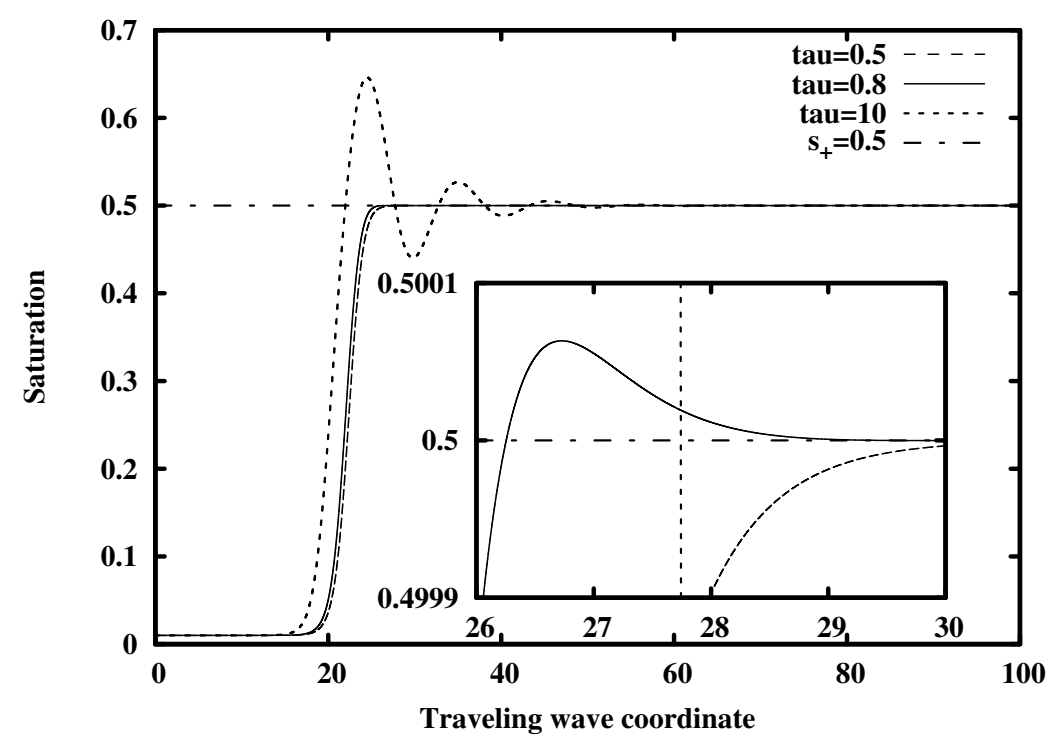

Figure 2: The saturation profiles obtained with parameters $D(s)=0.4, K(s)=s^{2}, s_{+}=0.5$, and different $\tau=0.5$, $0.8,10$.

In the following subsection, the numerical discretization is introduced firstly, then the description of a predictor-corrector process for resolving the nonlinearity of the governing equation follows.

\subsection{Discretization of the relaxation non-equilibrium Richards equation}

The variational form of NERE is to find $s \in L^{2}\left([0, T] ; H^{1}(\Omega)\right)$ such that

$$
\begin{aligned}
& \left(\frac{\partial s}{\partial t}, \varphi\right)+(D(s) \nabla s, \nabla \varphi)+\left(\tau K(s) \nabla \frac{\partial s}{\partial t}, \nabla \varphi\right)-\left(\frac{\partial K(s)}{\partial z}, \varphi\right) \\
= & 0, \quad \forall \varphi \in L^{2}\left([0, T] ; H^{1}(\Omega)\right),
\end{aligned}
$$

where $H^{1}(\Omega)=\left\{\varphi \in L^{2}(\Omega): \partial^{\alpha} \varphi \in L^{2}(\Omega)\right.$ for all $\left.|\alpha| \leq 1\right\}$ is the standard Sobolev space given in a bounded domain $\Omega \subset \mathcal{R}^{2}$. The boundary of $\Omega$, assumed piecewise sufficiently smooth, is denoted by $\partial \Omega$. We assume that the boundary $\partial \Omega$ is composed of two complementary parts $\partial \Omega=\partial \Omega_{D} \cup \partial \Omega_{N}$, where $\partial \Omega_{D}$ represents Dirichlet boundary and $\partial \Omega_{N}$ represents the Neumann boundary.

Let $\mathcal{I}=\left\{\left[t_{n-1}, t_{n}\right]: 1 \leq n \leq N_{\mathcal{I}}, 0=t_{0}<\cdots<t_{N_{\mathcal{I}}}=T\right\}$ define the time partitions. For every $n$ with $1 \leq n \leq N_{\mathcal{I}}$, we denote the $n$-th subinterval by $I_{n}=\left[t_{n-1}, t_{n}\right]$ with the length $\Delta t_{n}=t_{n}-t_{n-1}$. Moreover, we use $\mathcal{T}:=\left\{\mathcal{K}_{i}\right\}_{i=1}^{N_{\text {tot }}}$ to denote a triangulation which completely covers the domain $\Omega$, where $\mathcal{K}_{i}$ denotes the $i$-th element, and $N_{\text {tot }}$ is the total number of elements. Then the finite element space $V_{\mathcal{T}} \in H^{1}(\Omega)$ is addressed as

$$
V_{\mathcal{T}}=\left\{\varphi \in H^{1}(\Omega):\left.\varphi\right|_{\mathcal{K}} \in P_{l}(\mathcal{K}), \forall \mathcal{K} \in \mathcal{T}\right\},
$$


where $\mathcal{K}$ is an element of $\mathcal{T}$ and $P_{l}(\mathcal{K})$ denotes the set of polynomials with degree $\leq l$ over an element $\mathcal{K}$. We use the piecewise linear finite element to approximate the unknown $s$ in (3.1), i.e.,

$$
s_{\mathcal{T}}=\sum_{i=1}^{N_{\mathcal{T}}} s_{i} \varphi_{i}(\mathcal{T}),
$$

where $\left\{\varphi_{i}(\mathcal{T})\right\}_{i=1}^{N_{\mathcal{T}}}$ is the set of basis functions of the finite dimensional subspace $V_{\mathcal{T}}$, $\left\{s_{i}\right\}_{i=1}^{N_{\mathcal{T}}}$ is the set of coefficients of the unknown $s_{\mathcal{T}}$.

Dealing with the temporal discretization, we adopt the Crank-Nicolson method. For convenience, we define a sequence $\left\{s^{(k)}: k=0,1, \cdots, L\right\}$, where $L$ is a maximum iteration step, to denote $s^{n+1}$. To solve the non-linear Eq. (3.1), we use a predictor-corrector process in each time propagation step. The prediction of the numerical solution at the time instant $t_{n+1}$, i.e., $s^{(0)}$, is obtained by solving

$$
\frac{s^{(0)}-s_{\mathcal{T}}^{n}}{\Delta t_{n}}\left(\mathbb{M}+\tau K\left(s_{\mathcal{T}}^{n}\right) \mathbb{K}\right)+D\left(s_{\mathcal{T}}^{n}\right) \mathbb{K} s^{(0)}=\mathbb{F}\left(s_{\mathcal{T}}^{n}\right),
$$

while the correction $s^{(k)}$ from the $k$-th correction step is obtained by solving

$$
\begin{aligned}
& \frac{1}{2} \frac{s^{(k)}-s_{\mathcal{T}}^{n}}{\Delta t_{n}}\left(\mathbb{M}+\tau K\left(s^{(k-1)}\right) \mathbb{K}+\mathbb{M}+\tau K\left(s_{\mathcal{T}}^{n}\right) \mathbb{K}\right) \\
= & \frac{1}{2}\left(\mathbb{F}\left(s_{\mathcal{T}}^{n}\right)-D\left(s_{\mathcal{T}}^{n}\right) \mathbb{K} s_{\mathcal{T}}^{n}+\mathbb{F}\left(s^{(k-1)}\right)-D\left(s^{(k-1)}\right) \mathbb{K} s^{(k-1)}\right),
\end{aligned}
$$

where $\mathbb{M}$ and $\mathbb{K}$ are matrices, $\mathbb{F}$ is a vector, which can be written as follows

$$
\begin{aligned}
& \mathbb{M}_{i, j}=\int_{\Omega} \varphi_{i}(\mathcal{T}) \varphi_{j}(\mathcal{T}) d x d z \\
& \mathbb{K}_{i, j}=\int_{\Omega} \nabla \varphi_{i}(\mathcal{T}) \nabla \varphi_{j}(\mathcal{T}) d x d z, \\
& \mathbb{F}_{j}=\int_{\Omega} K^{\prime}(s) \frac{\partial s}{\partial z} \varphi_{j}(\mathcal{T}) d x d z
\end{aligned}
$$

From the above predictor-corrector process, a sequence $\left(s^{(k)}\right)$ will be generated from which we obtain $s_{\mathcal{T}}^{n+1}=s^{(k)}$ when $s^{(k)}$ and $s^{(k-1)}$ are close enough. In the implementation, we also introduce the maximum number $L$ so that $s_{\mathcal{T}}^{n+1}=s^{(L)}$ for balancing the efficiency and accuracy.

\subsection{An a posteriori error estimate for NERE}

It can be observed from Fig. 2 that the large variation on the saturation happens only around the wetting front. In fact, this is also the case in the simulations in the high dimensional spaces. Hence, $h$-adaptive mesh methods become a feasible way for improving the simulation efficiency. 
To realize the $h$-adaptive method in an efficient way, a quality data structure and a well-designed error indicator are necessary. For the data structure, we follow [18] to use the hierarchy geometry tree to manage the mesh. The hierarchy geometry tree in two dimensional space is designed based on the fork tree, on which we actually can efficiently resolve a belonging-to relation between two elements from two different meshes, respectively. This belonging-to relation allows an efficient implementation for the interpolation of the numerical solutions between two different meshes, which guarantees the efficient implementation for the $h$-adaptive process during the simulations. In the following, we introduce the design of the error indicator, which is also a very important component for a quality $h$-adaptive method.

Briefly, the error indicator of the numerical solution in each element consists of three components, i.e., the jump of the gradient of the saturation across the edge of the element, the local residual of the governing from the numerical solution, as well as the difference of the saturations from the adjacent time instants.

Specifically, error indicator of our algorithm can be described in detail as follows. Suppose that the current time instant is $t_{n}$, and we have obtained the prediction $s^{(0)}$ for the saturation at the time instant $t_{n+1}$ by solving (3.4). Then the element residual for the element $\mathcal{K}$ is defined by

$$
R_{1, \mathcal{K}}=\frac{s^{(0)}-s_{\mathcal{T}_{n}}^{n}}{\Delta t_{n}}-\nabla \cdot D\left(s_{\mathcal{T}_{n}}^{n}\right) \nabla s_{\mathcal{T}_{n}}^{n}-\frac{\partial K\left(s_{\mathcal{T}_{n}}^{n}\right)}{\partial z}-\tau \nabla \cdot\left(K\left(s_{\mathcal{T}_{n}}^{n}\right) \nabla\left(\frac{s^{(0)}-s_{\mathcal{T}_{n}}^{n}}{\Delta t_{n}}\right)\right)
$$

We also define the jump

$$
\mathcal{J}_{1, e}=n_{e} \cdot \nabla s_{\mathcal{T}_{n}}^{n}, \quad e \in E,
$$

where $E$ denotes the collection of the element edges, $n_{e}$ is the unit outer normal. With these notations, a residual space-time error estimator for the NERE is given by

$$
\eta_{1, \mathcal{K}}^{n}=\left\{\Delta t_{n} h_{\mathcal{K}}^{2}\left\|R_{1, \mathcal{K}}\right\|_{\mathcal{K}}^{2}+\sum_{e \in E} \Delta t_{n} h_{e}\left\|\mathcal{J}_{1, e}\right\|_{e}^{2}+\Delta t_{n}\left\|s^{(0)}-s_{\mathcal{T}_{n}}^{n}\right\|_{\mathcal{K}}^{2}\right\}^{\frac{1}{2}},
$$

where $\eta_{1, \mathcal{K}}^{n}$ is a local error indicator on the element $\mathcal{K},\|\cdot\|$ denotes the $H^{1}$-norm, $h_{\mathcal{K}}$ and $h_{e}$ denote for the sizes of the element $\mathcal{K}$ and the edge $e$, respectively. The term $\left\|s^{(0)}-s_{\mathcal{T}_{n}}^{n}\right\|$ can be regarded as the temporal error indicator. The error indicator $\eta_{1, \mathcal{K}}^{n}$ is used to do the mesh refinement after the prediction step.

Similarly, the residual under the Crank-Nicolson scheme is written as

$$
\begin{aligned}
R_{2, \mathcal{K}}= & \frac{s_{\mathcal{T}_{n+1}}^{n+1}-s_{\mathcal{T}_{n+1}}^{n}}{\Delta t_{n}}-\frac{1}{2} \nabla \cdot\left(D\left(s_{\mathcal{T}_{n+1}}^{n}\right) \nabla s_{\mathcal{T}_{n+1}}^{n}+D\left(s_{\mathcal{T}_{n+1}}^{n+1}\right) \nabla s_{\mathcal{T}_{n+1}}^{n+1}\right) \\
& -\frac{1}{2} \frac{\partial}{\partial z}\left(K\left(s_{\mathcal{T}_{n+1}}^{n}\right)+K\left(s_{\mathcal{T}_{n+1}}^{n+1}\right)\right) \\
& -\frac{\tau}{2} \nabla \cdot\left(\left(K\left(s_{\mathcal{T}_{n+1}}^{n}\right)+K\left(s_{\mathcal{T}_{n+1}}^{n+1}\right)\right) \nabla\left(\frac{s_{\mathcal{T}_{n+1}}^{n+1}-s_{\mathcal{T}_{n+1}}^{n}}{\Delta t_{n}}\right)\right) .
\end{aligned}
$$


The jump contains both $s_{\mathcal{T}_{n+1}}^{n}$ and $s_{\mathcal{T}_{n+1}}^{n+1}$ and is written as

$$
\mathcal{J}_{2, e}=n_{e} \cdot \nabla\left(\frac{s_{\mathcal{T}_{n+1}}^{n}+s_{\mathcal{T}_{n+1}}^{n+1}}{2}\right)
$$

With the above two quantities the corresponding error estimator is defined as

$$
\eta_{2, \mathcal{K}}^{n}=\left\{\Delta t_{n} h_{\mathcal{K}}^{2}\left\|R_{2, \mathcal{K}}\right\|_{\mathcal{K}}^{2}+\sum_{e \in E} \Delta t_{n} h_{e}\left\|\mathcal{J}_{2, e}\right\|_{e}^{2}+\Delta t_{n}\left\|s_{\mathcal{T}_{n+1}+1}^{n+1}-s_{\mathcal{T}_{n+1}}^{n}\right\|_{\mathcal{K}}^{2}\right\}^{\frac{1}{2}}
$$

As mentioned previously, in the predictor-corrector process, the mesh will only be adaptively refined in the prediction step, this is a nonlinear process from $t_{n}$ to $t_{n+1}$. During this process, we have to keep the old solution and have to correct the new solution for several times. We locally refine the elements with error indicators $\eta_{1, \mathcal{K}}^{n}$ larger than adaption tolerance while keeping other elements unchanged to obtain the quality mesh $\mathcal{T}_{n+1}$. With this strategy, it can keep the mesh fitting the old solution and the new solution, which means the process can guarantee the numerical accuracy well. Besides, solution $s_{\mathcal{T}_{n+1}}^{n+1}$ is obtained by predictor-corrector process. This procedure can be summarized as the Algorithm 3.1.

$\overline{\text { Algorithm 3.1 Algorithm for only refinement of mesh and the predictor-corrector pro- }}$ cess.

Input: $s_{\mathcal{T}_{n}}^{n}$ with mesh $\mathcal{T}_{n}$ on its time interval $I_{n+1}, k=1$, maximum iteration step $L=3$.

Output: A quality mesh $\mathcal{T}_{n+1}$ and $s_{\mathcal{T}_{n}+1}^{n+1}$.

1: Solve Eq. (3.4) to predict a solution $s^{(0)}$ on the mesh $\mathcal{T}_{n}$.

2: According $s_{\mathcal{T}_{n}}^{n}$ and $s^{(0)}$ generate the error indicator $\eta_{1, \mathcal{K}}^{n}$ by formula (3.8).

3: According to the $\eta_{1, \mathcal{K}}$ only refine the mesh $\mathcal{T}_{n}$ to obtain mesh $\mathcal{T}_{n+1}$.

4: Projecting $s_{\mathcal{T}_{n}}^{n}$ and $s^{(0)}$ onto the mesh $\mathcal{T}_{n+1}, s_{\mathcal{T}_{n+1}}^{n}$ and $s_{\mathcal{T}_{n+1}}^{(0)}$ are obtained correspondingly.

5: while $k \leq L$ and err $>10^{-9}$ do

6: $\quad$ Solve Eq. (3.5) to obtain $s_{\mathcal{T}_{n}+1}^{(k)}$,

$$
s_{\mathcal{T}_{n+1}}^{n}, s_{\mathcal{T}_{n+1}}^{(k-1)} \longrightarrow s_{\mathcal{T}_{n}+1}^{(k)}
$$

7: $\quad$ estimate $\operatorname{err}=\left\|s_{\mathcal{T}_{n+1}}^{(k)}-s_{\mathcal{T}_{n+1}}^{(k-1)}\right\|_{2}$.

8: $\quad s_{\mathcal{T}_{n}+1}^{n+1}=s_{\mathcal{T}_{n}+1}^{(k)}$.

9: $\quad k=k+1$.

10: end while

11: return $\mathcal{T}_{n+1}$ and $s_{\mathcal{T}_{n}+1}^{n+1}$.

If the mesh grids increase only with the time evolution, the efficiency will slow down 
since there will be lots of mesh grids in the region with gentle solutions causing by the movement of the wetting front. We design the algorithm below to resolve this issue.

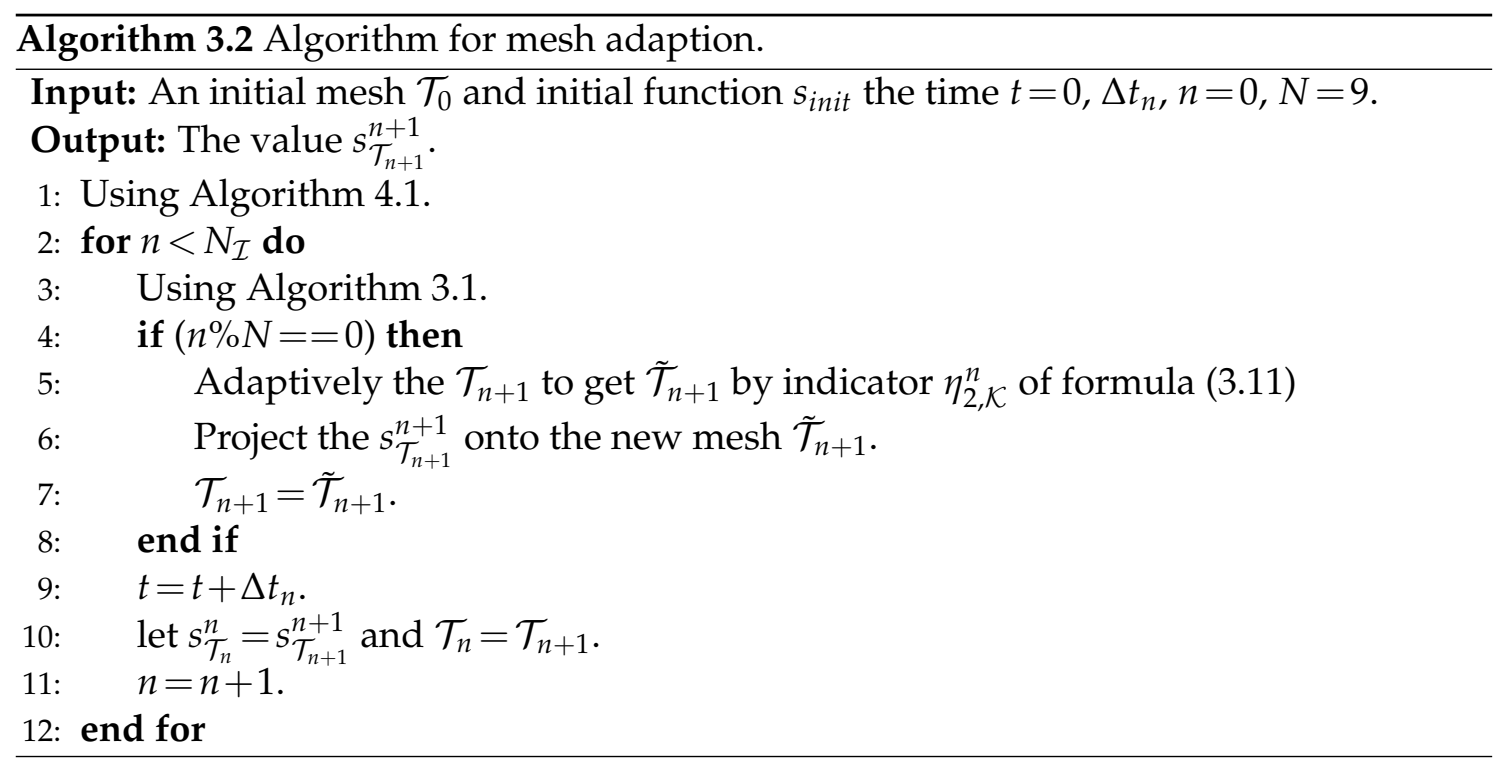

Since we are solving a temporal equation, and predictor-corrector process is employed to resolve the nonlinearity of the equation, the adaptive process in the algorithm is designed as follows. We assume that the numerical approximation $s_{\mathcal{T}_{n}}^{n}$ has been already solved on the current mesh $\mathcal{T}_{n}$ at the time instant $t_{n}$. In the predictor-corrector process, we use the forward Euler method to propagate the solution from $t_{n}$ to $t_{n+1}$, and the prediction of the saturation at $t_{n+1}$ is denoted by $\tilde{s}^{(0)}$. After that, we do the mesh refinement of the mesh $\mathcal{T}_{n}$ to get the mesh $\mathcal{T}_{n+1}$, based on the error indicator $\eta_{1, \mathcal{K}}^{n}$ from $s_{\mathcal{T}_{n}}^{n}$ and $\tilde{s}^{(0)}$. Then, the solutions $s_{\mathcal{T}_{n}}^{n}$ and $\tilde{s}^{(0)}$ are transferred the finite element space built on $\mathcal{T}_{n}$ to the finite element space built on $\mathcal{T}_{n+1}$, by using the interpolation, so that $s_{\mathcal{T}_{n+1}}^{n}$ and $s^{(0)}$ are obtained correspondingly. For the correction process, details are showed as follows. First, we use the solutions $s_{\mathcal{T}_{n+1}}^{n}$ and $s^{(0)}$ to get the solution $s_{\mathcal{T}_{n+1}}^{n+1}$ by Crank-Nicolson scheme (3.5), and then we update $s^{(0)}$ with $s_{\mathcal{T}_{n+1}^{n+1}}^{n+1}$ to get $s^{(1)}$. Second, we repeat to solve Equation (3.5) and update $s^{(k)}$ with $s_{\mathcal{T}_{n+1}^{n+1}}^{n+1}$ to get $s^{(k+1)}$ by increasing the integer number $k$, until the error between $s_{\mathcal{T}}^{n+1}$ and $s^{(k)}$ satisfies the criterion of the correction step or $k$ is big enough.

It is noted that even the implementations on the adaptive refinement of the mesh and the solution interpolation are efficient with the help of C++ library AFEPack [18], the process can still occupy non-ignorable CPU time.

In the $h$-adaptive process, the mesh grids will be added locally in the region with large error indicator, and the mesh grids will be removed locally in the region with small error indicator. It can be observed in the simulation that the mesh grids will be added and removed repeatedly in some region around the wetting front, due to the dynamics of 
the wetting front. Hence, a strategy is used in our simulation to accelerate the implementation, i.e., we implement the adaptive refinement of the mesh grid by only adding the mesh grids, and implement the adaptive coarsening of the mesh grids by only removing the mesh grids every $N$ time steps, for a given $N$. With this strategy, the issue mentioned above can be avoided effectively.

\section{Numerical results}

In this section, three kinds of numerical experiments are delivered. Firstly, the numerical convergence of the $h$-adaptive method is tested by the pseudo-analytical solutions. Secondly, the advantage of $h$-adaptive method is demonstrated by simulating a single finger, i.e., the wetting front is resolved well by a number of mesh grids and the mesh density is small in other region. Thirdly, several parameters are tested in the formation of the fingers, and the width of the finger is studied by several experiments with different width of the channels.

\subsection{Preparation of the initial mesh}

For the initial condition, the wetting front always be a sharp one. Hence, it is necessary to design a quality nonuniform mesh to resolve the initial condition well.

In this subsection, a quality mesh $\mathcal{T}_{0}$ is given by adapting the initial coarse partition $\mathcal{T}_{\text {ini }}$ with the following error indicator

$$
\left\|s_{\mathcal{T}_{\text {ini }}}^{0}-s_{\text {init }}\right\|_{2}, \quad \mathcal{K} \in \mathcal{T}_{\text {ini }},
$$

where $s_{\mathcal{T}_{\text {ini }}}^{0}$ is finite element function by projecting initial saturation function $s_{\text {init }}$ on to mesh $\mathcal{T}_{\text {ini }}$. According to the given error indicator, the mesh grids will be added and removed repeatedly in some region around the wetting front. The details are showed in Algorithm 4.1.

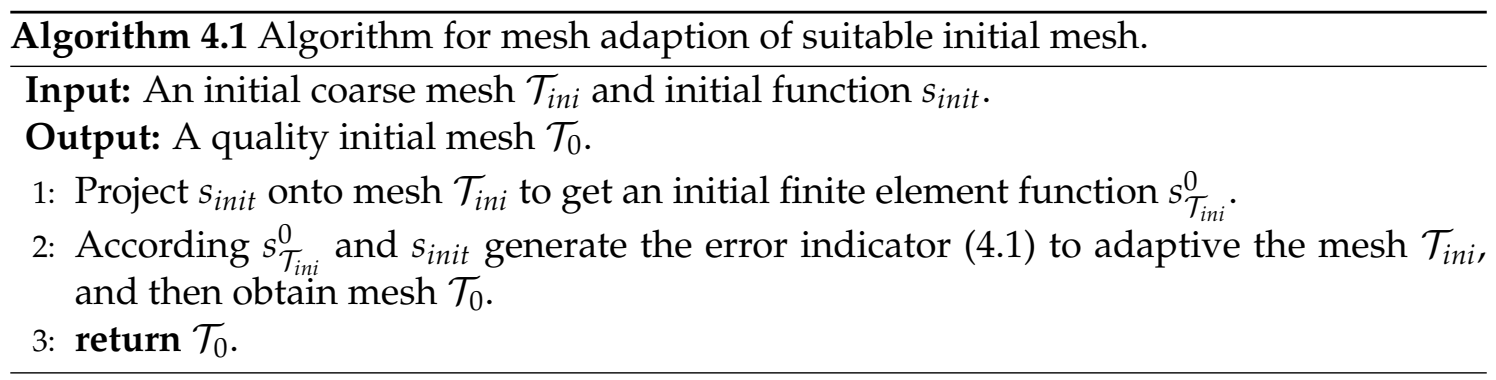

\subsection{Numerical convergence}

In this example, the rectangle $[0,2] \times[0,1000]$ computational region and corresponding boundary conditions are showed in Fig. 1. Based on the analysis in Subsection 2.2 and 


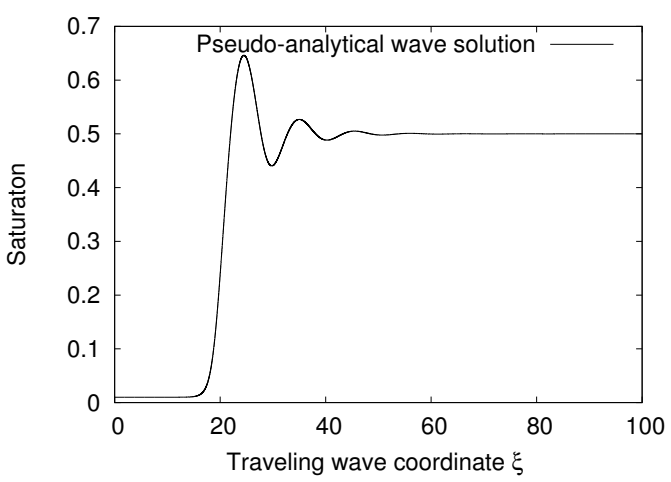

(a)

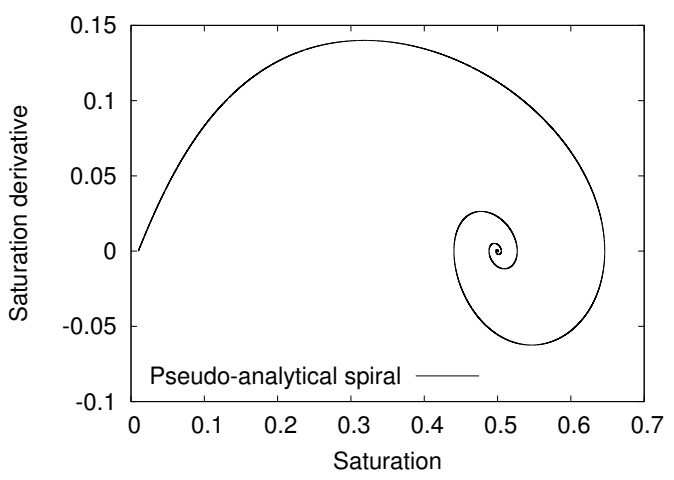

(b)

Figure 3: Pseudo-analytical solution of the ODE system (2.11) with parameters $D(s)=0.4, K(s)=s^{2}, s_{+}=0.5$, $s_{-}=0.01$ and $\tau=10$. (a) the wave solution. (b) the wave solution in the phase field.

numerical simulations in the Fig. 2, we consider $\tau=10$ in the example. Other parameters $D(u)=0.4, K(u)=u^{2}, s_{+}=0.5$, and $s_{-}=0.01$ are used. In the phase field, the stationary point $\left(s_{+}, 0\right)$ is connected by saddle point $\left(s_{-}, 0\right)$, in the right figure of Fig. 3 . The connection also coincides with the previous works [28,31,33].

The ODE System (2.11) is solved by using fourth-order Runge-Kutta schemes. The pseudo-analytical wave solution, which is showed in the left one of Fig. 3, presents the saturation overshoot. The value of saturation of the wetting front is up to around 0.65. The pseudo-analytical wave solution in the phase plane, which is showed in the right one of Fig. 3, presents the spiral. The spiral consists with the theoretical analysis in Subsection 2.2. Then, we use the pseudo-analytical solution in the phase plane as a reference solution.

Next, we solve Eq. (2.9) using the Algorithm 3.2, and the parameters are same to the ODE system, with the Dirichlet boundary condition

$$
s_{0}=0.5 \text {, }
$$

and initial condition

$$
s_{\text {init }}=0.245 \times \tanh (z-997)+0.255 .
$$

The numerical results are obtained at $t=100$ with step size $d t=0.01$.

In Fig. 4, the pseudo-analytical solutions, the finite element solutions on three successively and uniformly refined mesh, as well as the $h$-adaptive finite element solutions on three adaptively refined meshes are demonstrated. The left column of Fig. 4 shows the comparison of reference solution and the finite element solutions on the uniform meshes, while the right column shows the comparison of the reference solution and the $h$-adaptive finite element solutions. Two observations can be made from these figures. First, the numerical convergence can be observed successfully from both columns, i.e., 

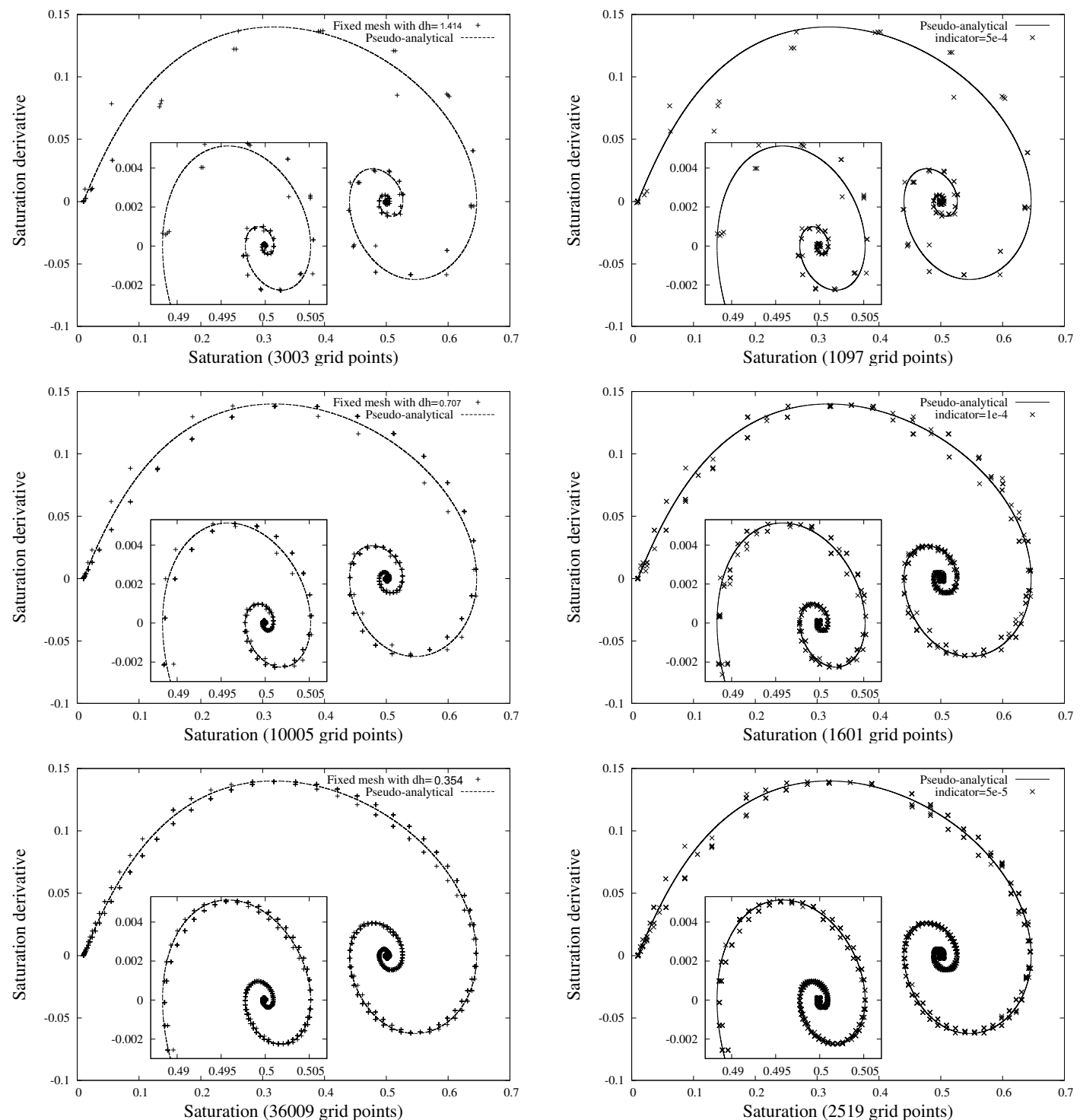

Figure 4: The pseudo-analytical solution noted solid line and the numerical solution noted points. The left column are the solutions with fixed uniform mesh, and right column are the solutions with the $h$-adaptive mesh technique.

with the increase of mesh grids the finite element solution agrees with the reference solution better and better. Second, our $h$-adaptive method successfully saving the computational resources, i.e., the $h$-adaptive result with 2519 mesh grids is comparable, or even better than that on the uniform mesh with 10005 mesh grids. 


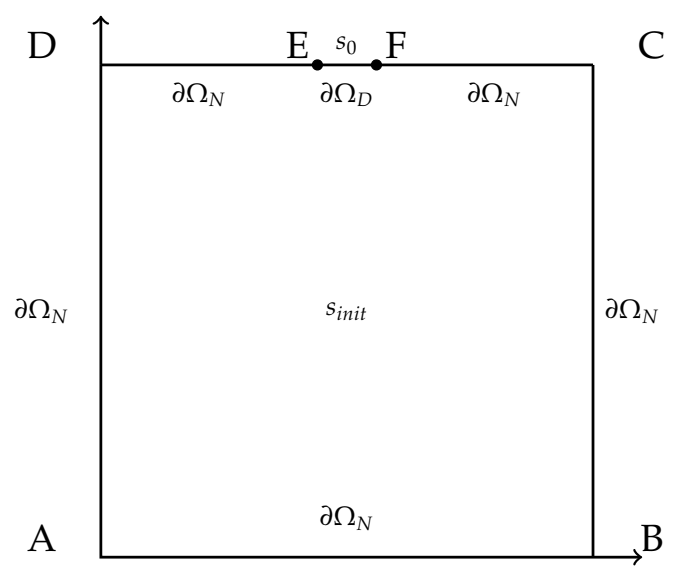

Figure 5: The computational region $[0,5] \times[0,5]$ and the boundary condition of single finger.

\subsection{Simulations of a single finger}

In this subsection, we use the following parameters $K(s)=s^{3}, \Psi(s)=-s^{\frac{-1}{4}}$, and $s_{+}=0.5$ to test the performance of our $h$-adaptive method on simulating a single finger. According to the theoretical analysis in Subsection 2.2 with $s_{-}=0.05, \tau_{c r i}$ is given by 0.0843 . The numerical results showed in Fig. 6(a) for different $\tau$. By numerical results, the saturation overshoot phenomenon can be observed obviously when $\tau=0.5$. With the fixed $\tau=0.5$, different values of $s_{-}$, for example $s_{-}=0.02,0.06,0.1$, influence on the saturation profiles of the saturation. The results are showed in Fig. 6(b). The consistent results to [16] are observed, i.e., with smaller $s_{-}$, the larger saturation on the finger tip is obtained, and the wetting front becomes sharper.

In the following, we show the feature of $h$-adaptive finite element method. In this example, the computational domain is showed in Fig. 5. The Dirichlet boundary condition $s_{0}=0.6$ is used for the segment EF i.e., $x \in[2.2,2.8]$ on the boundary $z=5$, and homogeneous Neumann boundary conditions are used for the other boundaries. Different initial saturations are used in the dry region, $s_{\text {init }}=s(x, z)+0.1, s_{\text {init }}=s(x, z)+0.06$, and $s_{\text {init }}=s(x, z)+0.02$, where

$$
s(x, z)=0.1 \tanh (2(z-4.8)) \tanh (2(5.2-z)+1) \tanh (2(x-2.2)) \tanh (2(2.8-x)+1),
$$

to show the efficiency of $h$-adaptive method.

In Fig. 7, the left one is the saturation distribution with $s_{\text {init }}=s(x, z)+0.1$, the middle one is the coarse initial mesh $\mathcal{T}_{\text {ini }}$. Since the initial mesh is too coarse to catch the initial saturation well, the Algorithm 4.1 is used to prepare the initial quality mesh, which is given in Fig. 7(b). As the rate of solution change rapidly from the wetting front to drying phase, our $h$-adaptive method adopts a "refine when required" strategy to catch the rapid change. The improvement of the mesh grids can be seen clearly, compare with the initial coarse one (Fig. 7(c)). 


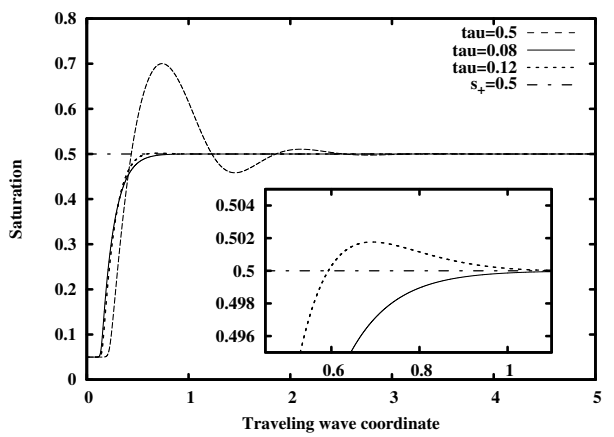

(a)

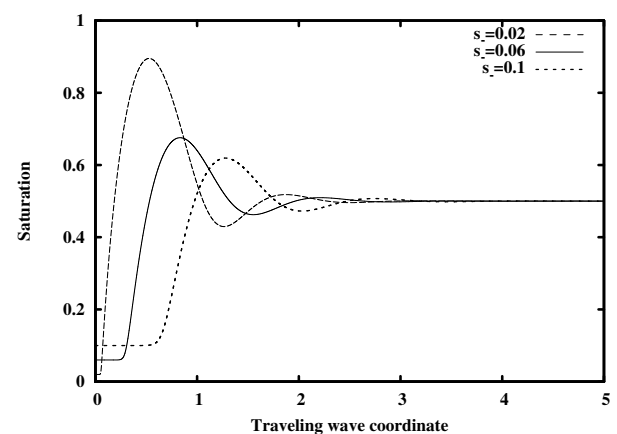

(b)

Figure 6: Pseudo-analytical solution of the ODE system (2.11) with $s_{+}=0.5$ and $D(s)=K(s) \Psi^{\prime}(s)$, where $K(s)=s^{3}, \Psi(s)=-s^{-1 / 4}$. (a) the saturation obtained with $s_{-}=0.05$ and different $\tau$, and (b) the saturation obtained with $\tau=0.5$ and different $s_{-}$.

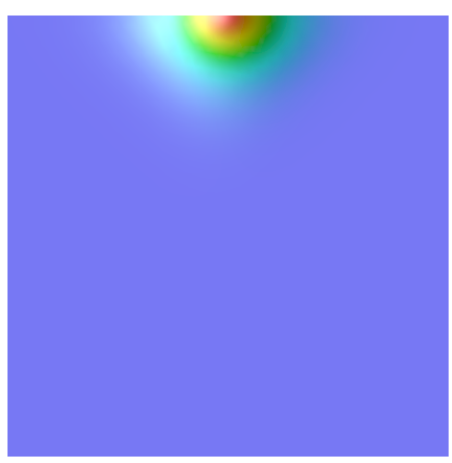

(a)

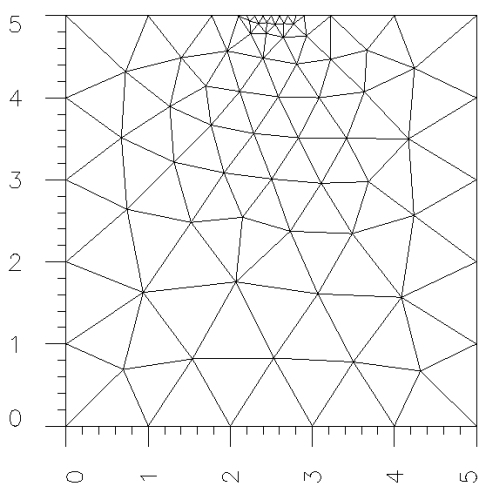

(b)

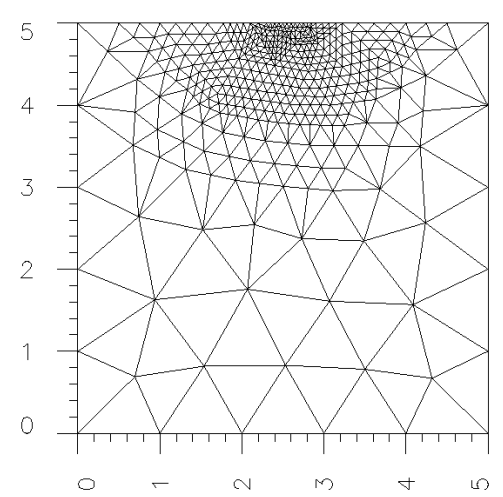

(c)

Figure 7: (a) the initial value $s_{\text {init. }}$ (b) the initial coarse mesh. (c) The prepared suitable initial mesh. 

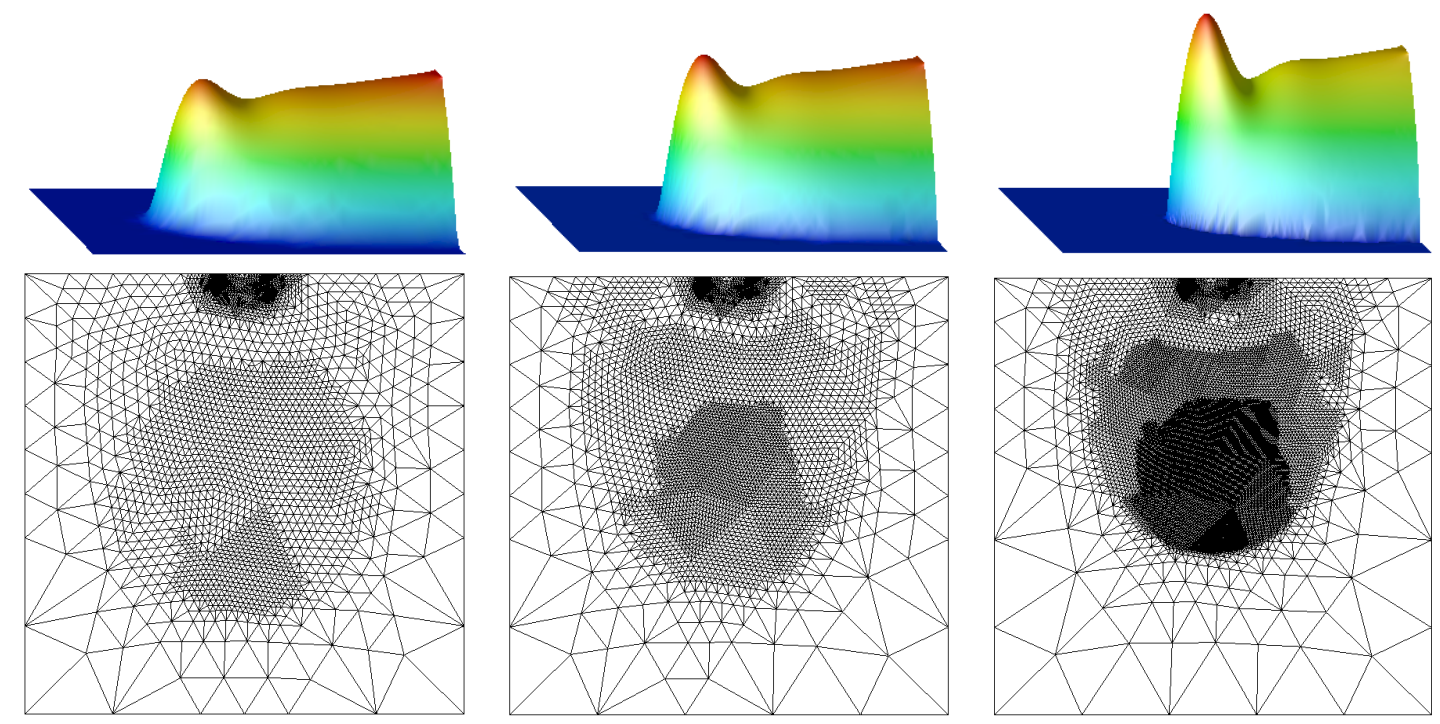

Figure 8: The NERE solution by using $h$-adaptive method shows at $t=12$ with the parameters: $K(s)=s^{3}$, $\Psi(s)=-s^{-1 / 4}, \tau=0.5, s_{+}=0.5$. On the top row, the saturation distribution is showed. On the bottom row, the corresponding $h$-adaptive mesh is showed. There are three columns. The first column shows the result with $s_{-}=0.1$, the second column shows the result with $s_{-}=0.06$, and the third column shows the result with $s_{-}=0.02$.

The side views of the finger at $t=12$ are showed in the right column of Fig. 8 by using $h$-adaptive finite element method. The saturation of drying phase $s_{-}$is $0.1,0.06$, and 0.02 from left to right, respectively. In these three simulations, the same tolerance of error indicator $10^{-4}$ for mesh adaption is used. Since the rate of solution change at the wetting front become bigger with decreasing $s_{-}$, the number of mesh points become larger from top to bottom. Hence, these numerical simulations show that the $h$-adaptive method is good at seizing the wetting front.

The results from Fig. 8 well matches the pseudo-analytical solution, which is showed in the right of Fig. 6. Besides, in Subsection 2.2, we get the velocity of traveling wave coordinate $v=\left(K\left(s_{+}\right)-K\left(s_{-}\right)\right) /\left(s_{+}-s_{-}\right)$. When $s_{-}$is smaller, the velocity of infiltration of flow is smaller by given $K(s)$ and $s_{+}$. From Fig. 6 , we can see the length of single finger becomes shorter, when the saturation of drying phase $s_{-}$becomes smaller, which verifies the velocity.

\subsection{Simulations of the formation process of the fingers}

In this subsection, we use $h$-adaptive finite element method to check the formation of the fingers by NERE model and predict the finger width numerically by changing the width of the computational domain.

The configuration of the numerical experiments is as follows. First of all, the compu- 


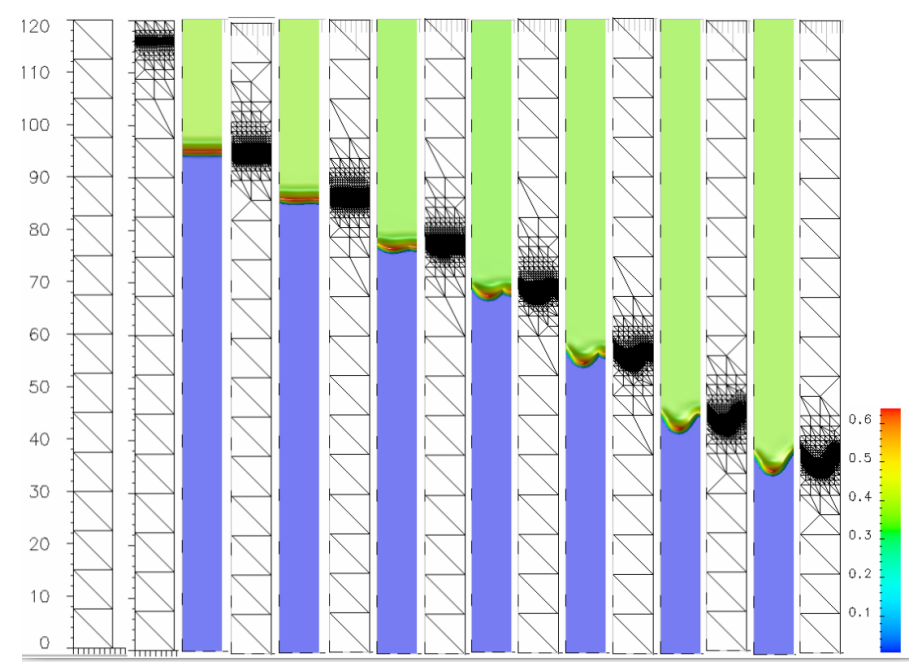

Figure 9: The dynamics of the wetting front by solving the NERE solution with $h$-adaptive method on the domain $[0,7.5] \times[0,120]$. The following parameters are used, i.e., $K(s)=s^{2}, D(s)=s^{2}, \tau=1, s_{+}=0.4$ and $s_{-}=0.01$. From left to right, the first two figures show the coarse initial mesh and the well prepared initial mesh, while the following figures appear in pair, in which the left one shows the saturation and the right one show the corresponding mesh, respectively. The time instants are $t=20,55,77,99,120,150,199$, respectively.

tational domain is given by Fig. 1 , with $A D=120$, and two different widths, i.e., $A B=7.5$, 30. In the following simulations, even though the width of the computational domain is changed, the number of the mesh grid is still in charge of the error indicator and gathers around the wetting front. The Dirichlet boundary condition $s_{0}=0.4$ works on the boundary $C D$, while the homogeneous Neumann boundary conditions are used for the other boundaries. The initial condition is consistent with given by

$$
s(x, z, 0)=0.195 \times \tanh (40 \times(z-116))+0.205 .
$$

In the simulations, we always use $\tau=1, d t=0.01, K(s)=s^{2}, D(s)=s^{2}$, and tolerance for the mesh adaptivity is $10^{-3}$. From the initial condition, we can see that there is a distribution layer [21] on the top of the domain. The quality initial mesh is prepared with Algorithm 4.1 to catch the distribution of initial saturation. Then the wetting front moves downward with the time evolution. Because of the instability of the governing equation, numerical error from the numerical method, as well as the perturbation along the transverse direction of the wetting front appears. With the time evolution, the perturbation grows and the structure of the finger formed. The above process can be observed successfully in Fig. 10, in which the initial perturbation appears around $t=77$, and four fingers are formed clearly around $t=199$. The advantage of the $h$-adaptive method can be seen obviously, i.e., a nonuniform mesh is used to describe the movement and variation of the wetting front.

From numerical results of Fig. 9 and Fig. 10, the flows arrive at around $z=35$ at $t=199$, i.e., the velocity of flows is about 0.407 . The theoretic velocity is 0.41 , which means 


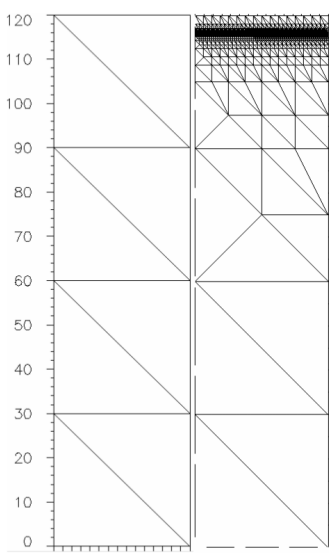

$t=0$

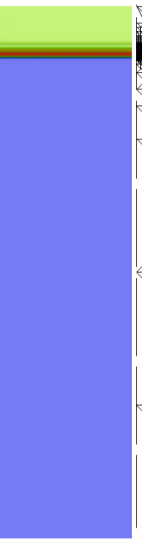

$t=20$

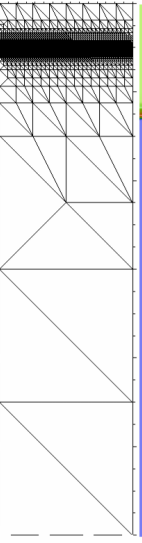

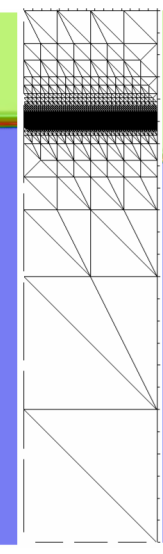

$t=55$

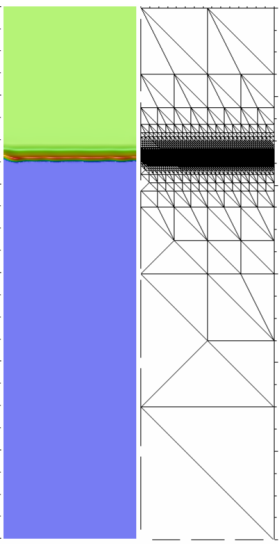

$t=77$

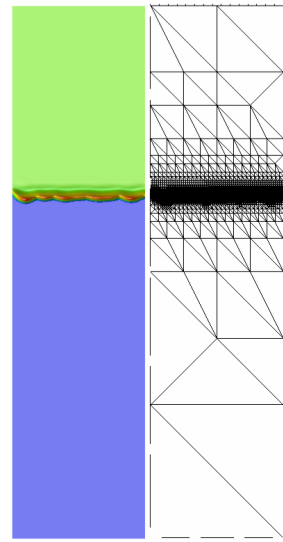

$t=99$

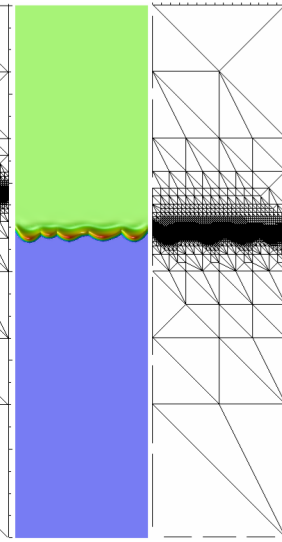

$t=120$

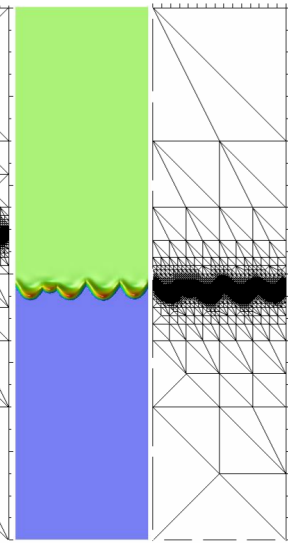

$t=150$

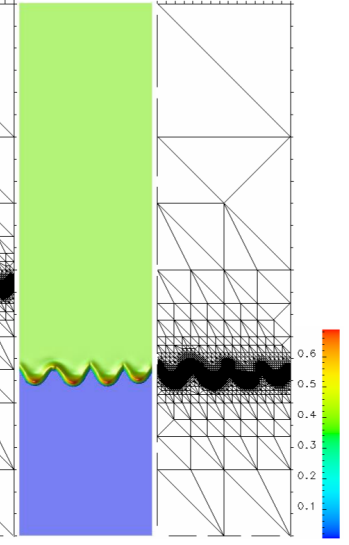

$t=199$

Figure 10: The dynamics of the wetting front by solving the NERE solution with $h$-adaptive method on the domain $[0,30] \times[0,120]$. The parameters used here are same to the ones in Fig. 9. The demonstration is also same to the one in Fig. 9.

the velocity of flows is not related with the width of computational domain. However, the formation of fingers needs longer time with the width of the computational width widen. Since the NERE model is short of the static hysteresis mechanism [24], which can limit the fluid spreading in the lateral, the formation of fingers becomes difficult. As a consequence, it is difficult to keep the separation of fingers for NERE model, i.e., the small fingers can not be longer once fingers form.

In this example, we confirm the NERE model can form fingers. To a certain extent, we confirm that NERE cannot describe fingering phenomenon very well $[3,24]$, since these fingers can not separate well. The formation of fingers appears as a winner-takesall process, by which the fastest growing fingers in the initial unstable front channelize 
most of the infiltrating fluid and inhibit the growth of other incipient fingers $[4,13,27]$. From Fig. 9 and Fig. 10, the tiny and later formed finger is suppressed until it disappears. Besides, we give a rough estimate of the width of fingers around 7 . When the width of the computational domain is 30 , there is four fingers at $t=199$.

\section{Conclusions}

In this paper, we numerically study the NERE by using an $h$-adaptive finite element method. The standard linear element and predictor-corrector method and Crank-Nicolson scheme are used to discretize the governing equation spatially and temporally, respectively. The numerical convergence of the $h$-adaptive finite element method has been demonstrated in experiments with three successively refined meshes. With the help of $h$-adaptive mesh method, a large amount of grid points is clustered around the wetting front. So the instability of the NERE is detected very well, which has been shown clearly in the numerical experiments.

It is found in the literature that the static and dynamic hysteresis is the key mechanism behind the fingering phenomenon in the porous medium flows. With the proposed effective numerical method in this paper, the static and dynamic hysteresis will be numerically studied with NERE in our future work.

\section{Acknowledgements}

The authors would like to thank Nianyu Yi from Xiangtan University and Hong Zhang from National University of Defense Technology for their valuable discussions. The research is partially supported by FDCT of Macao SAR (FDCT 029/2016/A1), MYRG of University of Macau (MYRG2017-00189-FST, MYRG2019-00154-FST), National Natural Science Foundation of China (Grant Nos. 11922120, 11871489, and 11401608), and Department of Science and Technology of Guangdong Province.

\section{References}

[1] J. BEAR, Dynamics of Fluids in Porous Media, Courier Corporation, 2013.

[2] A. Yu Beliaev And S. M. Hassanizadeh, A theoretical model of hysteresis and dynamic effects in the capillary relation for two-phase flow in porous media, Transport in Porous Media, 43(3) (2001), pp. 487-510.

[3] M. CHAPWANYA AND J. M. STOCKIE, Numerical simulations of gravity-driven fingering in unsaturated porous media using a nonequilibrium model, Water Resources Res., 46(9) (2010).

[4] L. Cueto-Felgueroso AND R. JuAnes, Nonlocal interface dynamics and pattern formation in gravity-driven unsaturated flow through porous media, Phys. Rev. Lett., 101 (2008), 244504.

[5] L. Cueto-Felgueroso And R. JuAnes, A phase field model of unsaturated flow, Water Resources Res., 45(10) (2009). 
[6] D. A. DiCARLO, Capillary pressure overshoot as a function of imbibition flux and initial water content, Water Resources Res., 43(8) (2007).

[7] D. A. DiCARLO, Stability of gravity-driven multiphase flow in porous media: 40 years of advancements, Water Resources Res., 49(8) (2013), pp. 4531-4544.

[8] H. DONG, Z. QIAO, S. SUN, AND T. TANG, Adaptive moving grid methods for two-phase flow in 415 porous media, J. Comput. Appl. Math., 265 (2014), pp. 139--150.

[9] A. G. Egorov, R. Z. DAutov, J. L. Nieber, AND A. Y. SHEShuKOV, Stability analysis of traveling wave solution for gravity-driven flow, in S. Majid Hassanizadeh, Ruud J. Schotting, William G. Gray, and George F. Pinder, editors, Computational Methods in Water Resources, volume 47 of Developments in Water Science, pages 121-128, Elsevier, 2002.

[10] A. G. Egorov, R. Z. Dautov, J. L. Nieber, AND A. Y. SHeSHukOV, Stability analysis of gravity-driven infiltrating flow, Water Resources Res., 39(9) (2003).

[11] M. ELIASSI AND R. J. GLASS, On the porous-continuum modeling of gravity-driven fingers in unsaturated materials: Extension of standard theory with a hold-back-pile-up effect, Water Resources Res., 38(11) (2002), pp. 16-1-16-11.

[12] W. FENG AND H. HUANG, Fast prediction of immiscible two-phase displacements in heterogeneous porous media with convolutional neural network, Adv. Appl. Math. Mech., 13(1) (2020), pp. 140162.

[13] R. J. GLAss, T. S. STEENHUIS, AND J.-Y. PARLANGE, Wetting front instability: 2. experimental determination of relationships between system parameters and two-dimensional unstable flow field behavior in initially dry porous media, Water Resources Res., 25(6) (1989), pp. 1195-1207.

[14] W. G. GRAY AND S. M. HASSANIZADEH, Unsaturated flow theory including interfacial phenomena, Water Resources Res., 27(8) (1991), pp. 1855-1863.

[15] S. M. HASSANIZADEH AND W. G. GRAY, Thermodynamic basis of capillary pressure in porous media, Water Resources Res., 29(10) (1993), pp. 3389-3405.

[16] G. HU AND P. A. ZEGELING, Simulating finger phenomena in porous media with a moving finite element method, J. Comput. Phys., 230(8) (2011), pp. 3249-3263.

[17] A. LAMACZ, A. RÄTZ, AND B. SCHWEIZER, A well-posed hysteresis model for flows in porous media and applications to fingering effects, Adv. Math. Sci. Appl., 21(2) (2011), pp. 33.

[18] R. LI, On multi-mesh h-adaptive methods, J. Sci. Comput., 24(3) (2005), pp. 321-341.

[19] R. LI, T. TANG, AND P. ZHANG, Moving mesh methods in multiple dimensions based on harmonic maps, J. Comput. Phys., 170(2) (2001), pp. 562-588.

[20] S. M. HASSANIZADEH AND W. G. GRAY, Toward an improved description of the physics of twophase flow, Adv. Water Res., 16(1) (1993), pp. 53-67. Research Perspectives in Hydrology.

[21] J. Nieber, A. Sheshukov, A. EgOrov, AND R. DAutOV, Non-equilibrium model for gravitydriven fingering in water repellent soils: Formulation and $2 d$ simulations, in Soil Water Repellency, pages 245-257, Elsevier, 2003.

[22] J. L. Nieber, R. Z. DAutov, A. G. EgOROV, AND A. Y. SHeshukOV, Dynamic capillary pressure mechanism for instability in gravity-driven flows; review and extension to very dry conditions, Transport in Porous Media, 58(1-2) (2005), pp. 147-172.

[23] G. J. J. M. Pieters, Stability and Evolution of Gravity-Driven Flow in Porous Media Applied to Hydrological and Ecological Problems, volume 65, 2004.

[24] A. RÄTZ AND B. SCHWEIZER, Hysteresis models and gravity fingering in porous media, ZAMMJ. Appl. Math. Mech., / Zeitschrift für Angewandte Mathematik und Mechanik, 94(7-8) (2014), pp. 645-654.

[25] B. SCHWEIZER, Hysteresis in porous media: Modelling and analysis, Interfaces and Free Boundaries, 19(3) (2017), pp. 417-448. 
[26] G. Scovazzi, A. Gerstenberger, And S. S. Collis, A discontinuous Galerkin method for gravity-driven viscous fingering instabilities in porous media, J. Comput. Phys., 233 (2013), pp. 373-399.

[27] J. SELKer, J. Y. PARLANGE, AND T. STEENHUIS, Fingered flow in two dimensions: 2. predicting finger moisture profile, Water Resources Res., 28(9) (1992), pp. 2523-2528.

[28] C. J. VAn Duijn, S. M. Hassanizadeh, I. S. POP, AND P. A. ZEGELING, Non-equilibrium Models for Two Phase Flow in Porous Media: the Occurence of Saturation Overshoots, 2013.

[29] J. C. YARZE AND M. P. CHASE, E. coli o157:h7 another waterborne outbreak!, Am. J. Gastroenterol, 95 (2000), pp. 1096.

[30] Y. YUAN, H. SONG, C. LI, AND T. SUN, An upwind mixed finite element method on changing meshes for positive semi-definite oil-water displacement of darcy-forchheimer flow in porous media, Adv. Appl. Math. Mech., 12(5) (2020), pp. 1196-1223.

[31] P. A. ZEGELING, An adaptive grid method for a non-equilibrium PDE model from porous media, J. Math. Study, 48(2) (2015), pp. 187-198.

[32] H. ZHANG AND P. A. ZEGELING, A moving mesh finite difference method for non-monotone solutions of non-equilibrium equations in porous media, Communi. Comput. Phys., 22(4) (2017), pp. 935-964.

[33] H. ZHANG AND P. A. ZEGELING, A numerical study of two-phase flow models with dynamic capillary pressure and hysteresis, Transport in Porous Media, 116(2) (2017), pp. 825-846.

[34] H. ZHANG AND P. A. ZEGELING, Simulation of thin film flows with a moving mesh mixed finite element method, Appl. Math. Comput., 338 (2018), pp. 274-289. 